\title{
Influence of micro-impurity on protein crystal growth studied by the etch figure method
}

\author{
Guoliang Dai ${ }^{\mathrm{a}, *}$, Xingyu Liu $^{\mathrm{a}}$, Gen Sazaki ${ }^{\mathrm{b}}$, Xiaogang Zhang ${ }^{\mathrm{c}}$ \\ a National Microgravity Laboratory, Institute of Mechanics, Chinese Academy of Sciences, No. 15 Bei Si Huan Xi Road, Beijing 100190, China \\ b The Institute of Low Temperature Science, Hokkaido University, N19-W8, Kita-ku, Sapporo 060-0819, Japan \\ ${ }^{c}$ Department of Chemistry, Renmin University of China, No. 59 Zhongguancun Road, Beijing 100872, China
}

\section{A R T I C L E I N F O}

Available online 19 September 2008

PACS:

61.72.Ff

61.72.sh

Keywords:

A1. Biocrystallization

A1. Etching

A1. Impurities

B1. Lysozyme

\begin{abstract}
A B S T R A C T
The investigation of the effect of micro impurity on crystal growth by optical microscopy has been validated. The results showed that the growth rate of a lysozyme crystal was affected even if the concentration of impurity of fluorescent-labeled lysozyme (abbreviation, F-lysozyme) was very small. Different concentrations of F-lysozyme had different effects on crystal growth rate. The growth rate decreased much more as F-lysozyme concentration increased. The density of incorporated F-lysozyme on different grown layers of a lysozyme crystal during crystal growth was obtained from the results of flat-bottomed etch pits density.
\end{abstract}

(c) 2008 Elsevier B.V. All rights reserved.

\section{Introduction}

Protein crystallization is the first step of structure analysis of protein crystals by X-ray or neutron diffraction. It is necessary to purify protein before its crystallization, because the impurities adsorbed on a crystal surface strongly affect the surface morphology [1], nucleation and growth kinetics [2,3], diffraction [4] and crystal quality [5,6]. However, even if a protein solution has been purified, such as in the case of a commercial protein solution, the concentrations of impurities in protein solution are still high compared with a purified inorganic material [7]. Moreover, the impurity molecules in a protein solution are difficult to be removed [8]. For example, in a protein solution, there still exists a small amount of protein molecules even after careful purification of protein solution. They are usually protein oligomer or homologous protein molecules with a similar structure of protein molecules. We call these molecules microimpurity. Micro-impurity molecules affect protein crystal quality although their concentration in protein solution is low. For example, the point defects caused by the adsorption of microimpurities on crystal surface strongly affect the crystal quality $[9,10]$. However, until now only a very small number of works focus on micro-impurity in protein crystallization. In order to

\footnotetext{
* Corresponding authors. Tel.: +8610 82544125, fax: +861082544096 (Guoliang Dai); Tel.: +861062516958, fax: +861062515601 (Xiaogang Zhang).

E-mail addresses: dspr@imech.ac.cn (G. Dai), zhang_xg@ruc.edu.cn (X. Zhang).
}

obtain a high-quality crystal for analyzing its structure, we have to know how micro-impurities affect the crystallization process.

The methods of investigating micro-impurity are, to some degree, different with popular ones of studying impurities with high concentrations. HPLC [2], electrophoresis [11,12], and fluorescence intensity [13] are the main methods. However, they have some limitations. For example, HPLC and electrophoresis are invasive methods and can only obtain qualitative results. The fluorescence intensity method can obtain quantitative results, but it is a time-consuming method and requires fluorescent concentration. The etch figure method, which is usually applied in inorganic material studies, has been applied in protein crystallization research recently. In 2004, Hondoh et al. [9,10] studied the surface morphology of lysozyme crystals after etching by AFM. They found three types of etch pits: flat-bottomed type, deep flatbottomed type, and point-bottomed type. Their results showed that in the case of lysozyme crystal grown from purified solution, the origin of flat-bottomed etch pits on a crystal surface was vacancies. However, in the case of crystals grown from commercial lysozyme solution, the flat-bottomed etch pits were caused by the adsorption of impurity molecules. They analyzed the adsorption kinetics of impurities from the results of shape and etch pits density (EPD).

We have tried to observe and determine flat-bottomed etch pits by the etch figure method using phase contrast microscopy. By the optic method, we have some advantages when compared with AFM, such as precise temperature control and non-invasive observation. According to the sequence of the three types of etch pits, we can find the flat-bottomed-type etch pits. Then we can 
obtain the density of impurities incorporated with a crystal according to the EPD of flat-bottomed etch pits. We should point out that the etch figure method is in particular suitable for microimpurity study, since etch pits usually merge when the concentration of impurity is high.

In this paper, we adopted fluorescent-labeled lysozyme (F-lysozyme) as a model impurity molecule. We added F-lysozyme into specially purified lysozyme solution, where a seed lysozyme crystal started to grow. We etched the newly grown layers of a crystal and studied the effect of micro-impurity on crystal growth process and the amount of incorporated impurity on different layers of a crystal.

\section{Materials and methods}

\subsection{Materials}

The solution used for the experiments contained $1.01 \mathrm{mM}$ HEWL (99.99\% purity, Maruwa Food Industries, Inc., Japan), F-lysozyme (with different concentrations in different experiments), $25 \mathrm{mg} / \mathrm{ml} \mathrm{NaCl}$, and $50 \mathrm{mM}$ sodium acetate ( $\mathrm{pH} 4.5$ ). F-lysozyme was labeled with a fluorescent label, tetramethylrhodamin-5-isotiocyanate, according to the method described in Ref. [8]. All of the agents used in the experiments were analytically pure and filtered with $0.22 \mu \mathrm{m}$ Millipore filter membrane before use. An inverted phase contrast microscope (Olympus, IX71) was used. Its vertical and lateral resolution is 0.005 and $0.5 \mu \mathrm{m}$, respectively. The temperature inside the observation cell is $\pm 0.1^{\circ} \mathrm{C}$. F-lysozyme concentrations used in experiments were $0,280,330,380,430$, and $480 \mathrm{nM}$.

\subsection{Methods}

Lysozyme seed crystals were obtained by the batch method at $20^{\circ} \mathrm{C}$. After the size of a seed crystal reached $200-300 \mu \mathrm{m}$, it was transferred to an observation cell. Height of the newly grown layers was several $\mu \mathrm{m}$. We observed the $\{110\}$ face of a tetragonal lysozyme crystal. Temperature inside the observation cell was 20 and $33^{\circ} \mathrm{C}$ under crystal growth and etching conditions, respectively. The seed crystal in the observation cell grew for 5-6h, and the etching process continued for $40-50 \mathrm{~min}$. The morphologies of the $\{110\}$ face of a lysozyme crystal were taken by a CCD camera automatically for every minute during the etching process.

We analyzed etching figures by the Image-Pro Express software. We chose an area $\left(241 \times 156 \mu \mathrm{m}^{2}\right)$ for analysis, inside which there were no micro-crystals, adsorbed particles, or big deep flatbottomed etch pits on a crystal surface. The chosen area for observation was far away from the corner and edge of the crystal. We distinguished flat-bottomed etch pits according to the sequence of appearance of etch pits [14] and their gray values. We measured the number of flat-bottomed etch pits.

\section{Results and discussion}

\subsection{Observation of etch pits by the optical method}

We observed the formation process of etch pits in situ by the phase contrast microscopy. The phase contrast microscope used in our experiments has a vertical resolution of $5 \mathrm{~nm}$, which was almost the same as the height of one elementary step of a lysozyme crystal. Therefore, we can easily find etch pits on a crystal surface. Thanks to the large enough observation area, the error of statistical results in our experiments was small. Moreover, the optic method used in this work can avoid the problems in AFM observations, such as the disturbance of tips of the cantilever, evaporation of protein solution, etc.

The impurity effect of F-lysozyme was obvious [8]. After comparison with the crystals grown from commercial lysozyme solution $(6 \times$ re-crystallization, containing $1.5 \%$ impurity molecules), purified lysozyme solution, and purified lysozyme solution containing micro-impurities (the maximum impurity concentration was $0.024 \%$ ), we found that the EPD were different, which are shown in Fig. 1(a)-(c), respectively. The EPD in Fig. 1(a) was 10 times more than that in Fig. 1(c). Moreover, etch pits easily merged under the former conditions than under the latter one. This demonstrated that the etch figure method could not be used under the condition of solution containing high-concentration impurities.

The etch pits appeared in the observation area 5-10 min after the etching process started. The etch pits elongated in the [ $\overline{1} 10]$ direction with the shape similar to 2D islands on a crystal surface. In Fig. 1(c), two types of etch pits were found in the observation area: one was flat-bottomed etch pits, and the other was deep flat-bottomed etch pits. Few of the point-bottomed etch pits were found. We found that the positions of flatbottomed and deep flat-bottomed etch pits were independent under our etching conditions. In the etching process, the first appeared etch pits were deep flat-bottomed ones, the number of which kept constant in the whole etching process. This result was the same as that of Hondoh et al. [9,10], showing that the observation of etch pits caused by micro-impurities using phase contrast microscopy was feasible. Moreover, we found that the number of flat-bottomed etch pits gradually increased with the etching time within $40 \mathrm{~min}$. However, there was suddenly an order of magnitude increase in the number of flat-bottomed etch pits after an etching time of $40 \mathrm{~min}$, indicating that the top surface of the seed crystal before growth started to be etched. Therefore, the number and positions of flat-bottomed etch pits at the etching time 40 min showed the adsorption results of F-lysozyme at the beginning of the crystal growth process in solution containing F-lysozyme.

We also observed the morphologies of the $\{110\}$ surface of the same lysozyme crystal after etching and growing for several times. We etched a lysozyme crystal at $33^{\circ} \mathrm{C}$ for $40 \mathrm{~min}$, then grew the crystal for $80 \mathrm{~min}$ at $20^{\circ} \mathrm{C}$ in order to let the newly appeared
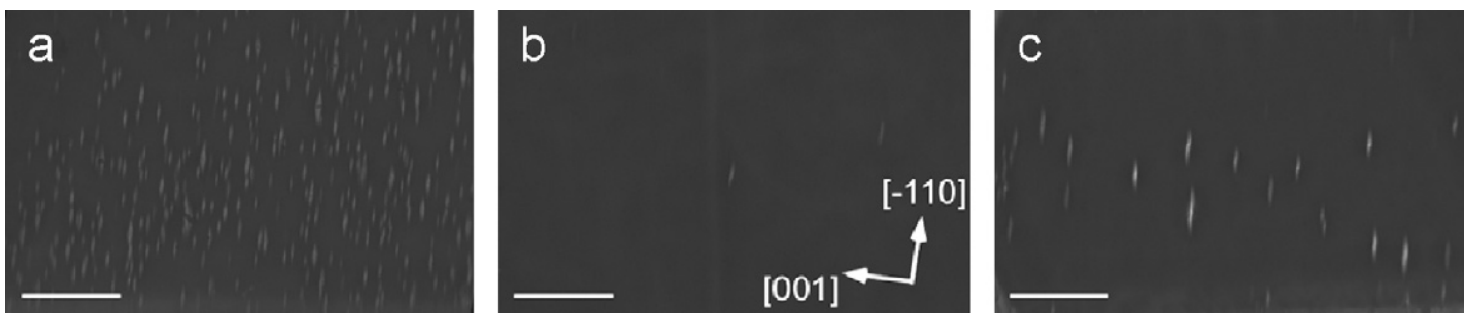

Fig. 1. Morphologies of the $\{110\}$ surface of the same lysozyme crystal grown from the different solutions after etching for 15 min. (a) Commercial lysozyme solution, (b) purified lysozyme solution, and (c) purified lysozyme solution containing $430 \mathrm{nM}$ F-lysozyme. The scale bar represents $25 \mu \mathrm{m}$. 

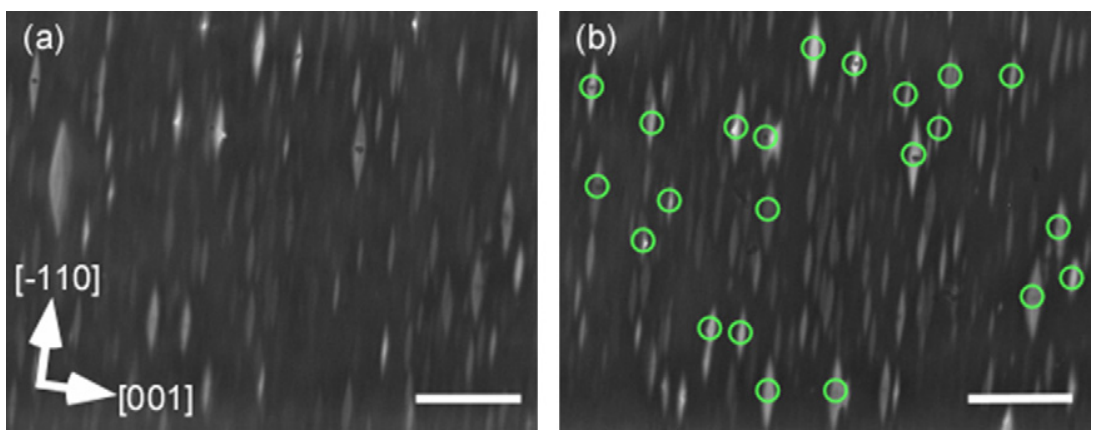

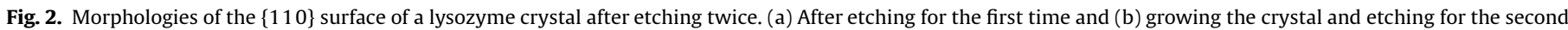
time. The etch pits appeared at the same positions in both etching processes were marked by $\circ$. The scale bar represents $25 \mu \mathrm{m}$.

a

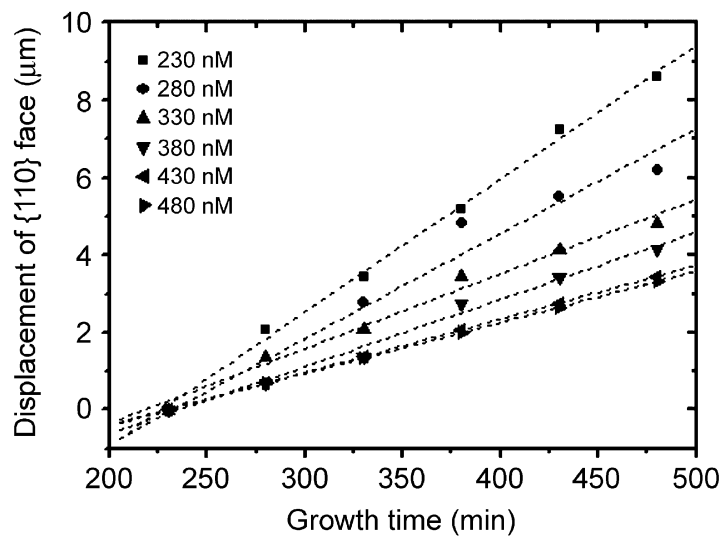

b

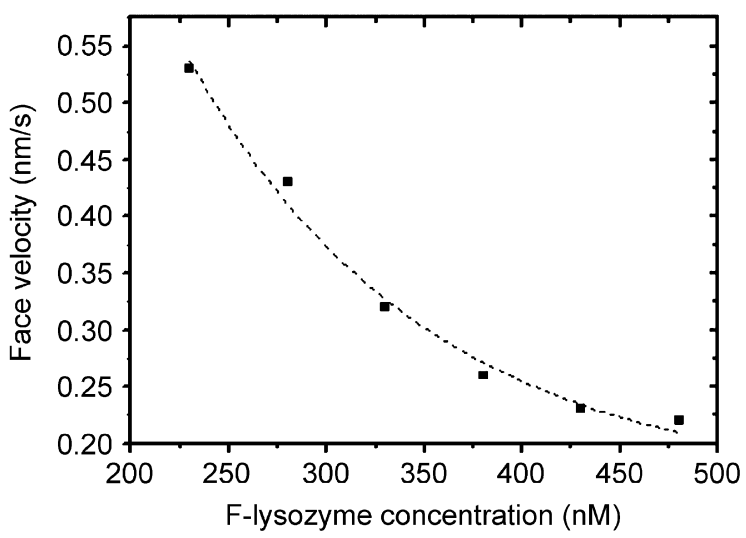

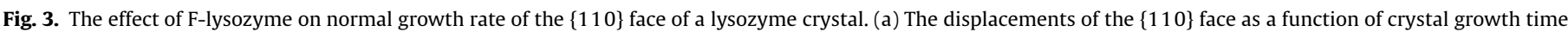

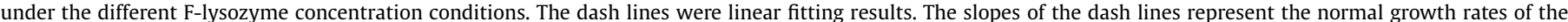
$\{110\}$ face and (b) the normal growth rate of the $\{110\}$ face as a function of F-lysozyme concentrations.

lysozyme layers cover all of the etch pits. Then, repeat the above procedure again for several times. The results were shown in Fig. 2. We found that the positions of most of deep flatbottomed and point-bottomed etch pits were the same during the etching and growth process, showing that the etch figure method could not eliminate the origin of deep flatbottomed and point-bottomed etch pits, and could not increase or decrease the number of them, as well. Therefore, the EPD of deep flat-bottomed and point-bottomed etch pits should keep constant during the etching process and most of the flatbottomed etch pits that gradually appeared in the etching process were caused by F-lysozyme (a small number of flatbottomed etch pits was caused by vacancies, which will be discussed in Section 3.3).

\subsection{Impurity effect on the normal growth rate of the $\{110\}$ face}

Fig. 3(a) showed the effect of F-lysozyme on the normal growth rate of the $\{110\}$ face of the crystal. F-lysozyme obviously decreased the normal growth rate of the $\{110\}$ face, and the higher the concentration of F-lysozyme, the smaller the normal growth rate. This indicated that the interaction between lysozyme molecules in solution and in crystal was hindered after the adsorption of F-lysozyme on a crystal surface. From Fig. 3(b), we found that the normal growth rate of the $\{110\}$ face exponentially decayed with the increase in F-lysozyme concentration, and the normal growth rate of the $\{110\}$ face became almost constant after the F-lysozyme concentration was higher than $430 \mathrm{nM}$. This result indicated that F-lysozyme had the maximum impurity effect on normal growth rate when its concentration was $430 \mathrm{nM}$ or higher.

The equilibrium adsorption model [15] assumes that the major limitation of growth rate comes from the impurity adsorption on a crystal surface. According to this model, the concentration of impurity molecules adsorbed on a crystal surface does not reach an equilibrium/saturated state under the condition of high normal growth rate of the crystal face, but it reaches the equilibrium/ saturated state when the normal growth rate of a surface is small. Considering the normal growth rate of the $\{110\}$ face was small and kept almost constant after the concentration of F-lysozyme was higher than $430 \mathrm{nM}$, we could conclude that the adsorption of F-lysozyme molecules on a crystal surface reached the equilibrium/saturated state when the concentration of F-lysozyme was higher than $430 \mathrm{nM}$.

Noriaki and Mullin [16] built a model to explain the impurity effect on growth rate of inorganic and organic crystals grown from solution. The equation is shown as follows:

$R / R_{0}=1-\lambda \theta_{\mathrm{eq}}=1-[\lambda K x /(1+K x)]$

where $R$ and $R_{0}$ are the normal growth rate of the crystal face under the conditions of no impurity and impurity, respectively. $\lambda$ is the ratio factor, $\theta_{\mathrm{eq}}$ is the cover degree of impurity molecules on a crystal surface, $K$ is the Langmuir constant, and $x$ is impurity concentration. 
The values of $\lambda$ and $K$ in our results were 1.18 and $0.00452 \mathrm{nM}^{-1}$, respectively, calculated by the non-linear leastsquare method according to Eq. (1). $\lambda=1.18$, which was much close to $\lambda=1$. According to the explanations of Kubota et al., the condition $\lambda=1$ showed that F-lysozyme molecules could easily adsorb onto the $\{110\}$ face, but could only have a weak affect on decreasing the normal growth rate of the $\{110\}$ face.

\subsection{The relationship between EPD and normal growth rate}

Flat-bottomed etch pits were caused by vacancies and impurity molecules. In order to obtain the density of incorporated F-lysozyme molecules, the contribution of vacancies should be deducted from the results of EPD. The relationship between the concentration of point defects and impurity was described by [17]

$N=N_{0} \exp (n C / T)$

where $N, N_{0}, C, n$, and $T$ are concentration of point defects, equilibrium concentration of point defects without impurity, constant, impurity concentration, and temperature, respectively.

As we have mentioned in Section 3.1, the results of EPD of flatbottomed etch pits at etching time $40 \mathrm{~min}$ revealed the incorporated F-lysozyme at the beginning of crystal growth. Then we calculated $N_{0}$ using the results of EPD as a function of F-lysozyme concentration (shown in Fig. 4, open circle) at etching time $40 \mathrm{~min}$, by the non-linear fitting method. The value of $N_{0}=0.4 \times 10^{-4} \mu \mathrm{m}^{-2}$, which was the number density of point defects caused by vacancies. From Fig. $4, N_{\min }$ and $N_{\max }$ were $3.5 \times 10^{-4}$ and $9.6 \times 10^{-4} \mu \mathrm{m}^{-2}$, respectively. Therefore, only $4-11 \%$ of flat-bottomed etch pits were caused by vacancies, which gave evidence of our viewpoint in Section 3.1 that "most of flatbottomed etch pits were caused by F-lysozyme molecules".

The relationship between EPD and normal growth rate of the $\{110\}$ face of a lysozyme crystal was investigated, which is shown in Fig. 5. It was indicated that the normal growth rate of the $\{110\}$ face of a crystal decreased with the increase in EPD value, i.e. increasing the density of incorporated F-lysozyme in a crystal. According to Eq. (3) [18]

$R=h / \tau$

where $h$ and $R$ are the height of steps and the normal growth rate of $\{110\}$ face, respectively. $\tau$ is the exposure time of the sites where impurity molecules adsorbed.

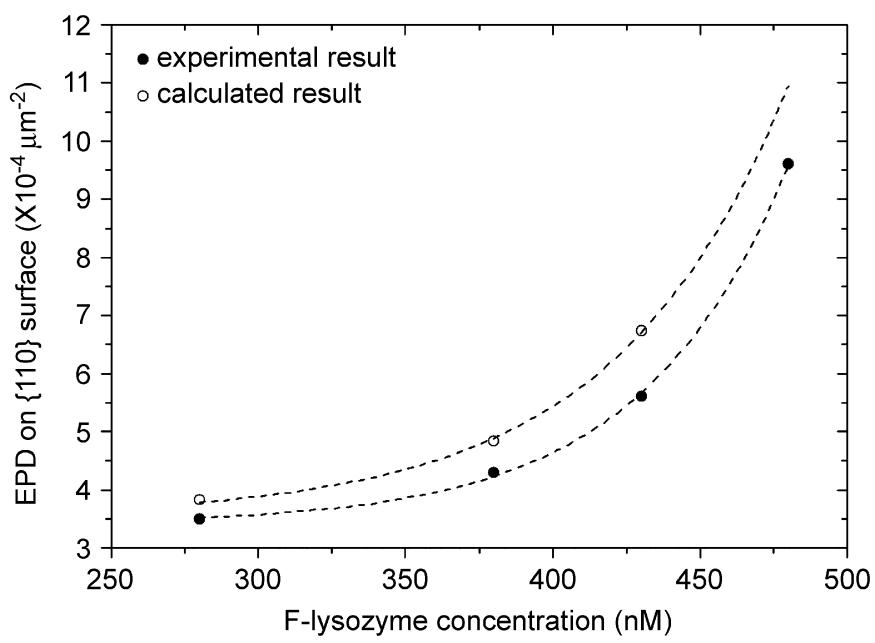

Fig. 4. EPD of flat-bottomed etch pits on a $\{110\}$ face of a lysozyme crystal after etching for $40 \mathrm{~min}$ as a function of the F-lysozyme concentration. Closed and open circles are experimental and calculated results, respectively.

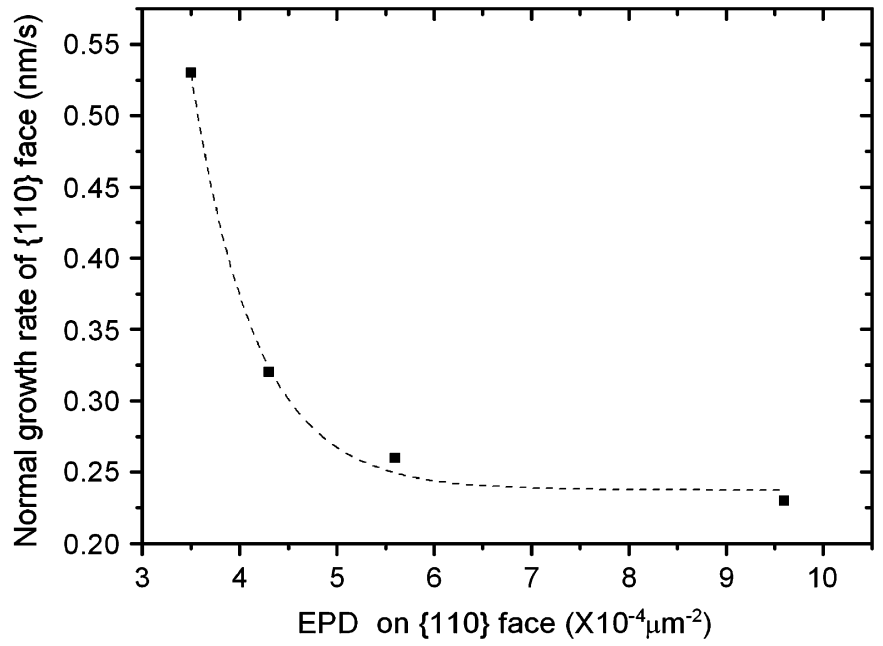

Fig. 5. Normal growth rate of a $\{110\}$ face as a function of EPD of flat-bottomed etch pits on a $\{110\}$ face of a lysozyme crystal.

Our results indicated that the exposure time of the sites where F-lysozyme incorporated with a crystal increased as the concentration of F-lysozyme increased.

After fitting exponential decay (first-order) of the data in Figs. 3(b) and 5, and fitting exponential growth of the data in Fig. 4 (open circle), we obtained the following equations:

$v=2.47 \exp (-c / 122)=0.16$

$\mathrm{EPD}=0.0004 \exp (c / 49.58)=3.4$

$v=60 \exp (-\mathrm{EPD} / 0.66)+0.24$

where $v$ represents normal growth rate of the $\{110\}$ face of a lysozyme crystal and $c$ represents F-lysozyme concentration.

According to Eqs. (4)-(6), we can obtain the calculated EPD result as a function of F-lysozyme concentration, which is shown in Fig. 4 (open circle). The calculated value of EPD was higher than the experimental one under the same F-lysozyme concentration condition. We could not distinguish the monomers or aggregates of F-lysozyme by phase contrast microscopy. Fig. 4 demonstrated that not only F-lysozyme monomer but also some aggregates of F-lysozyme adsorbed on the $\{110\}$ face. Therefore, from the EPD of flat-bottomed etch pits, the density of incorporated F-lysozyme could be obtained including the density not only of monomers but also of aggregates.

\subsection{The density of incorporated F-lysozyme in different grown layers}

We studied the changes of EPD of flat-bottomed etch pits under different F-lysozyme concentration conditions. Fig. 6(a) showed the values of EPD as a function of etching time under the conditions of F-lysozyme concentrations 330 and $430 \mathrm{nM}$. The etching process is an adverse process of growth process and the flat-bottomed etch pits were mainly caused by incorporated Flysozyme; therefore, we could deduce the density of incorporated F-lysozyme in different grown layers of a crystal. The EPD of flatbottomed etch pits at a growth time $t$ can be expressed as $\mathrm{EPD}_{t}=\sum_{t=0}{ }_{0} \mathrm{EPD}_{t}$. After we measured the EPD of newly appeared flat-bottomed etch pits at a growth time $t$, together with the normal growth rate and displacements of the $\{110\}$ face, we could calculate the density of incorporated F-lysozyme in different grown layers of a crystal. Fig. 6(b) shows the density of incorporated F-lysozyme at different grown layers of a crystal during the crystal growth process. This result revealed that the density of incorporated F-lysozyme in grown layers exponentially 
a

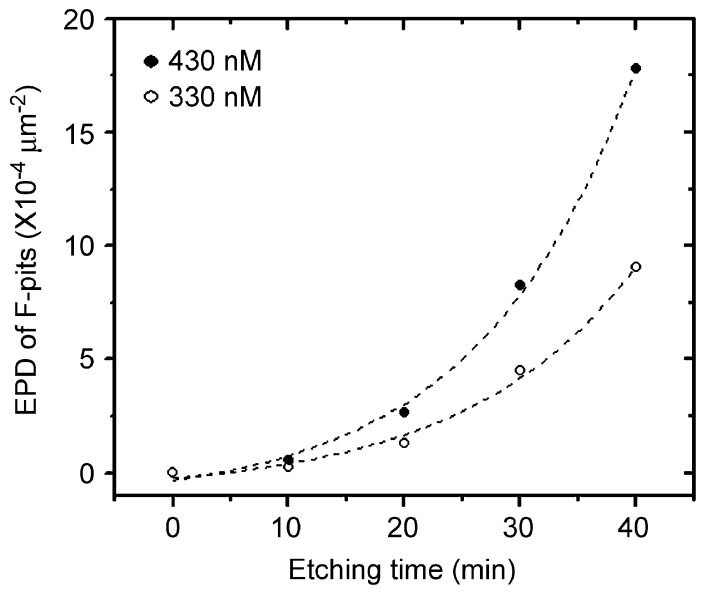

b

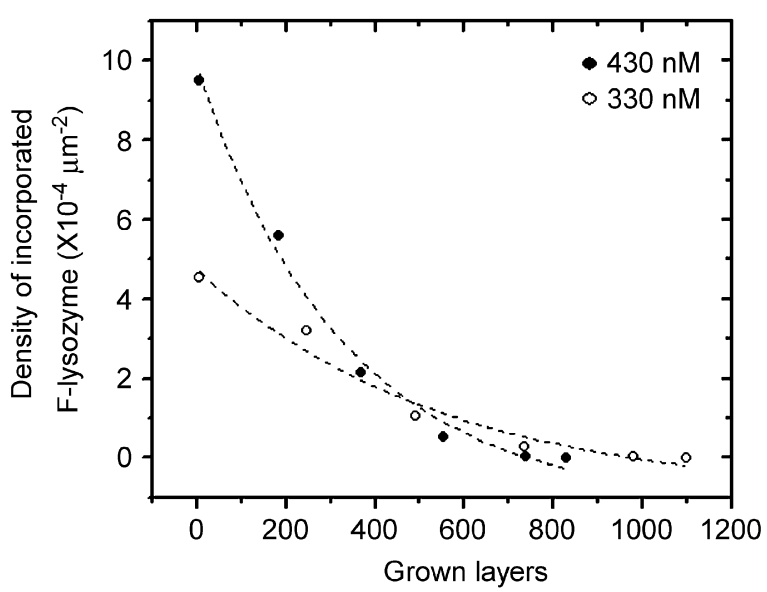

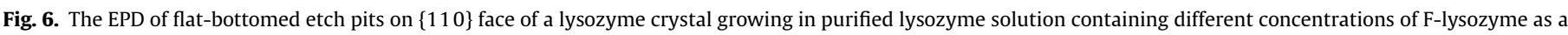
function of etching time (a), and density of incorporated F-lysozyme in grown layers of a lysozyme crystal (b).

decreased as a function of crystallization time. Moreover, this result also showed that micro-impurities had a maximum impurity effect at the beginning of the crystallization process, and the higher the concentration of micro-impurity, the greater the effect of them.

According to the Bruton-Prim-Slichter model [19], distribution coefficient of impurity molecules $k_{0}$ determines the distribution of impurity molecules around a crystal during the diffusion process of impurity molecules from the solution to a crystal surface. When $k_{0}>1$, the impurity distribution will gradually decrease from the center to the surface of a crystal. Our results of the density of incorporated F-lysozyme in different layers were similar to the results in Ref. [20] obtained by the fluorescence method, showing that the etch figure method enables us to consider the impurity incorporation behavior in detail.

\section{Conclusions}

The etch figure method together with phase contrast microscopy can be used in studying the effect of micro-impurity on the protein crystal growth process. The flat-bottomed etch pits on a crystal surface were almost caused by the impurity molecules (including monomers and aggregates) adsorbed on a crystal surface. The normal growth rate of a crystal surface decreased even if the impurity concentration was very small. The incorporated micro-impurity inside a crystal exponential decreased as a function of crystal growth time.

\section{Acknowledgements}

The authors thank grants from the National Nature Science Foundation of China (Nos. 20603043, 10432060, and 20576140).

\section{References}

[1] C. Abergel, M.P. Nesa, J.C. Fontecilla-Camps, J. Crystal Growth 110 (1991) 11.

[2] B.R. Thomas, P.G. Vekilov, F. Rosenberger, Acta Crystallogr. D 54 (1998) 226.

[3] J. Hirschler, J.C. Fontecilla-Camps, J. Crystal Growth 171 (1997) 559.

[4] C.L. Caylor, et al., Proteins 36 (1999) 270.

[5] I. Yoshizaki, A. Kadowaki, Y. Iimura, N. Igarashi, S. Yoda, H. Komatsu, J. Synchrotron Radiat. 11 (2004) 30.

[6] K. Izumi, S. Sawamura, M. Ataka, J. Crystal Growth 168 (1996) 106

[7] B.R. Thomas, P.G. Vekilov, F. Rosenberger, Acta Crystallogr. D 52 (1996) 776.

[8] T. Matsui, G. Sazaki, H. Hondoh, Y. Matsuura, T. Nakada, K. Nakajima, J. Crystal Growth 293 (2006) 415

[9] H. Hondoh, T. Nakada, Jpn. J. Appl. Phys. 43 (2004) 4529

[10] H. Hondoh, T. Nakada, J. Crystal Growth 275 (2005) e1423.

[11] M. Skouri, B. Lorber, R. Giegé, J.-P. Munch, J.S. Candau, J. Crystal Growth 152 (1995) 209.

[12] P.G. Vekilov, L.A. Monaco, B.R. Thomas, V. Stojanoff, F. Rosenberger, Acta Crystallogr. D 52 (1996) 785.

[13] K. Kurihara, S. Miyashita, G. Sazaki, T. Nakada, S.D. Durbin, H. Komatsu, T. Ohba, K. Ohki, J. Crystal Growth 196 (1999) 285.

[14] G. Sazaki, K. Tsukamoto, S. Yai, M. Okada, K. Nakajima, Cryst. Growth Des. 5 (2005) 1729.

[15] G.D. Botsaris, E.A. Mason, R.C. Reid, AIChE J. 13 (1967) 764.

[16] K. Noriaki, J.W. Mullin, J. Crystal Growth 152 (1995) 203.

[17] K. Tanahashi, M. Kikuchi, T. Higashino, N. Inoue, Y. Mizokawa, Physica B 273 (1999) 493.

[18] P.G. Vekilov, A. Mitsuo, K. Tatsuo, Acta Crystallogr. D 51 (1995) 207.

[19] J.A. Burton, R.C. Prim, W.P.J. Slichter, J. Chem. Phys. 21 (1953) 1987.

[20] I. Yoshikazu, I. Yoshizaki, H. Nakamura, S. Yoda, H. Komatsu, Cryst. Growth Des. 5 (2005) 301. 